\title{
Technology Makes the Heart Grow Fonder? A Test of Media Multiplexity Theory for Family Closeness
}

\author{
Bhoj Balayar * and Michael Langlais
}

Citation: Balayar, Bhoj, and Michael Langlais. 2021. Technology Makes the Heart Grow Fonder? A Test of Media Multiplexity Theory for Family Closeness. Social Sciences 10: 25. https://doi.org/10.3390/socsci 10010025

Academic Editor: Nigel Parton Received: 10 November 2020 Accepted: 8 January 2021 Published: 15 January 2021

Publisher's Note: MDPI stays neutral with regard to jurisdictional clai$\mathrm{ms}$ in published maps and institutional affiliations.

Copyright: (C) 2021 by the authors. Licensee MDPI, Basel, Switzerland. This article is an open access article distributed under the terms and conditions of the Creative Commons Attribution (CC BY) license (https:// creativecommons.org/licenses/by/ $4.0 /)$.
Department of Educational Psychology, University of North Texas, Denton, TX 76201, USA; mickey.langlais@gmail.com

* Correspondence: bhoj.balayar@unt.edu

\begin{abstract}
Technology can be helpful for family relationships. Media multiplexity theory illustrates that the more technological connections (i.e., multimodality) an individual has with their family members, the stronger that relationship. Yet, this theory assumes that spending time face to face (FtF) is equitable to multimodality for relationship quality. The goal of this study is to examine the impact of online and offline interactions for the quality of family relationships. Data are from undergraduate students completing an online survey $(\mathrm{N}=154)$. Results reveal that spending time $\mathrm{FtF}$ is better for the quality of relationships for one parent, but not the other. Although $\mathrm{FtF}$ was not significantly different for grandparent and sibling relationships, participants felt closer to siblings when they texted. Correlational analyses revealed that individuals scoring high in collectivism report that spending time FtF is associated with higher relationship closeness and love, particularly with parents, but not other family members. These results provide some evidence for the importance of spending quality time with family members, particularly for those who identify as collectivistic.
\end{abstract}

Keywords: media multiplexity theory; family closeness; collectivism

\section{Introduction}

The increased use of social media, texting, and video calling has changed the way many individuals communicate with their friends, family, and romantic partners. In many ways, technology has helped families strengthen their relationships, especially if they are not proximal. For instance, individuals can contact family members through texting, direct messages on social media, such as Facebook Messenger, and by viewing their family members' social media content, regardless of how close they live to each other. Media multiplexity theory (MMT; Haythornthwaite 2005) provides a theoretical rationale for why technology can strengthen family relationships. According to this theory, the more technological connections (i.e., multimodality) an individual has with their family members, the stronger that relationship. In other words, being Facebook friends with a family member and following a family member on Twitter or Instagram are different connections that can help strengthen a relationship. Additionally, this theory assumes that spending time face to face ( $\mathrm{FtF}$ ) is equitable to multimodality (Ledbetter 2015). Is this true? Does sending a message on social media or video chatting equate to spending face-to-face time with a family member? The goal of this study is to examine the impact of online and offline interactions for the quality of family relationships.

Additionally, it is possible that the influence of online and offline connections on family relationships varies by culture, particularly those from collectivistic backgrounds. People who identify as collectivistic are individuals whose focus is the family or the group, rather than the individual. Some studies have examined the role of culture, particularly collectivism and individualism for multimodality via MMT. For example, Barakji et al. (2018) examined how multimodality predicted family closeness in transnational families and found that increased technological connections was associated with more closeness, but the variety of connections was not significant for relationship quality. A rationale 
that these authors used to explain their findings was whether or not their participants scored high on collectivism, as technological connections contributed to family closeness for those whose focus was on the family (i.e., collectivistic) as opposed to the individual (i.e., individualistic). People who identify as individualistic may be less motivated to use technology to communicate and connect with family members, as their focus generally is on their own goals. Although this study advances understanding about collectivism and family closeness, more is needed regarding the varied ways that individuals can connect with family members (such as social media, texting, video calling, and FtF), across multiple family relationships. In order to test these ideas, we will examine how collectivism moderates the relationship between online and offline communication on relationship closeness across multiple family relationships: parents, grandparents, siblings, and aunts or uncles. The results of this study will help provide evidence for optimal ways to promote family relationships, which is crucial for individual and relational development.

\subsection{Prevalence of Technology within Families}

The use of technology has grown exponentially over the last two decades, particularly among young adults. In the U.S., the number of daily social media users is approximately seven-in-ten individuals. According to the Pew Research Center (2019), social media use was only 5\% in 2005 but increased to 72\% in February 2019. The four most popular social media platforms are Facebook, Instagram, Snapchat, and Twitter (Pew Research Center 2019). Instagram and Snapchat are particularly popular among adults between 18 and 29 years old, as usage rates are approximately $67 \%$ and $62 \%$, respectively. Facebook is popular for adults between the ages of 50 and 64 , with $68 \%$ using this platform (Perrin and Anderson 2019). Some of this growth in social media use can be explained by access to mobile media. For example, in 2011, only 35\% of the U.S. population had smart phone access, whereas, in 2019, the rate reached 81\% (Anderson 2019). Global social media use is also growing rapidly, from 2.65 billion users in 2018 to an expected 3.02 billion in 2021 (which is approximately $40 \%$ of the world population), with the Philippines ranking as the most involved in the world with time spent on social media (Clement 2019).

People often rely on technology to communicate with their immediate family members, friends, and romantic partners. Individuals can communicate with family members directly on social media, such as sending messages or snapchats, or indirectly, by posting and sharing content on their social media about their family or content directed at a family member. Individuals can also keep up with family members by viewing others' social media content, which is one way to stay in touch. Texting also provides a method in which individuals can communicate with family members. Some studies have demonstrated benefits of using technology in family relationships. For example, Williams and Merten (2011) found that technology facilitated family connectedness through social media, suggesting that the increased use of technology at home supports family connectedness and increases child-parent rapport.

In addition to these social media behaviors is virtual chatting, as individuals can use social media applications, such as WhatsApp or Facebook Portal, to video chat with others, including family members. In fact, most advertisements for video chatting are aimed at family relationships. Through the advancement of communication technology, such as video chatting, it is becoming easier and more common to maintain family relationships. People are using communication technology to connect with friends and family members when it is convenient for them, particularly for individuals with busy lives (Kennedy et al. 2008). For example, integrated media technology, which includes video chatting and texting, connects college students to parents when there are geographical restrictions, which commonly strengthens relationship quality (Ellison et al. 2007; Ruppel et al. 2018). Overall, there is support that media connections with family members are beneficial for those relationships. It seems logical that multiple connections are likely to assist relationship closeness. 
Although research shows the positive impact of multimodality for family relationships, there are also some studies that illustrate how technology negatively impacts family relationships. For example, family conflict increases in cases where time spent on technology replaces time spent in person with family members (Mesch 2006). Additionally, social media provides more opportunities to cyberbully or be cyberbullied by others, which can hinder individual and relational well-being (i.e., Valkenburg and Peter 2011), which could indirectly hinder individuals' relationships with their family. Family relationships can also be harmed if someone is addicted to technology or social media, as less time is spent with family members, increasing opportunities of conflict in addition to negatively impacting individual well-being (Dutot 2020). Taking this information together, it seems that multimodality can be beneficial for family relationships as long as it supplements face-to-face time together, rather than replacing family time, and when media is used wisely and responsibly.

\subsection{Media Multiplexity and Strengthening Family Relationships}

Media multiplexity theory (MMT; Haythornthwaite 2005) provides an explanation for how technology can be both good and bad for family relationships. The major focus of MMT is to employ multiple media to maintain interpersonal relationships. Multimodality provides methods for connecting people, expressing emotions, and promoting intimacy, including within family relationships. Greater multimodality strengthens relationship ties by providing multiple ways for individuals to communicate. This theory also argues for a reciprocal relationship between multimodality and family closeness, meaning that not only does being connected through multiple media improve the strength of a family relationship, but those in closer family relationships will also increase their number of media connections.

However, MMT views spending time $\mathrm{FtF}$ as another connection within a dyad, comparable to technological connections. For instance, Ledbetter (2015) stated that "both the face-to-face and online medium-specific constructs positively [predict the] strength of mutual influence" (p. 363) when discussing MMT. This raises the question about whether spending time $\mathrm{FtF}$ is equitable to multimodality for family relationships. Although this debate is not new in the context of romantic relationships, with FtF interactions being rated more highly than multimodality (i.e., Jamieson et al. 2020), more research is needed to understand how offline and online behaviors contribute to the quality of family relationships, particularly as the reviewed literature illustrated mixed findings for family closeness. Literature on mutual self-disclosure provides evidence for the importance of $\mathrm{FtF}$ interactions compared to online interactions. These studies emphasize the importance of reciprocal communication and perceived responsiveness, which is commonly missing in online interactions (Finkel et al. 2012; Laurenceau et al. 2004; Sproull et al. 1986). People generally prefer to bond in person than over texting or online messaging (Sherman et al. 2013). Based on this literature, we propose the following research question (RQ1):

$\mathrm{RQ}$ 1: Is spending time FtF more associated with relationship closeness than interactions via multimodality?

\subsection{Collectivism Orientation and Media Multiplexity}

Over the past three decades, the worldwide immigration rate has increased. In 2019, the number of immigrants reached nearly 272 million, where it was 153 million in 1990 (United Nations: Department of Economic and Social Affairs 2019). Among the worldwide immigration population, the U.S. has reported 51 million international immigrants in 2019 (United Nations: Department of Economic and Social Affairs 2019). Many individuals immigrating to the U.S. come from collectivistic cultures, whereas many individuals residing in the U.S. are individualistic (Hamamura 2012). As a result of these demographic changes, many individuals in the U.S. are likely to identify as either collectivistic and/or individualistic. Although it is possible to identify as both individualist 
and collectivistic, the majority of individuals usually align more closely with one end of the spectrum (Hofstede 2001).

Accordingly, it is likely that collectivism will influence how individuals use technology in their family relationships. An individualistic cultural orientation considers people as unique and autonomous actors who are guided by an individual goal. In a collectivistic cultural orientation, actors are interdependent with their social interests and groups where actors sacrifice their self-interests for the betterment of the group (Hofstede 2001). Family is the smallest unit in the collectivist cultural society and all members are socially integrated into their group. The individualist culture views the individual as the primary unit (Hofstede 2001).

While MMT was founded on the ties between people within a group (Haythornthwaite 2002), the motivation for using technology involves both self and social interests. Some individuals may be more focused on using technology for their own self-interests, which would be an individualistic orientation, whereas others may use technology primarily to maintain close relationships, which would be a collectivistic orientation. The influence of these orientations on family closeness, however, has received limited attention. An exception is a study by Barakji et al. (2018) which examined the relationship between cultural orientation and relational closeness in long distance family relationships. The main result of this study suggested that relational closeness increased in collectivistic cultures the more they communicated, regardless of the medium in which they communicated (Barakji et al. 2018). Because MMT does not account for collectivism or individualism, this theory assumes that these orientations are influenced the same way by multimodality, which may not be true. Rather, individualistic individuals may see media multimodality as helpful for relational development, but collectivistic individuals may not see this approach as helpful compared to spending time FtF. In an individualistic orientation, people may be more reliant on multimodality (compared to FtF) to feel close to their family, whereas those with a collectivistic orientation may not need multimodality as much as individualistic individuals do to maintain their relationships, but rather FtF time would be more beneficial. Based on this information, we believe that collectivism will moderate the relationship between multimodality, face-to-face time together, and relationship quality, which is answered by research question 2 (RQ2):

RQ2: How does collectivism moderate the relationship between multimodality, spending time FtF, and relationship closeness?

\section{Methods}

\subsection{Participants}

College students were recruited from undergraduate human development and family science courses at a large university in the southern central U.S. Approximately 154 students agreed to participate in this study. The average age of participants was $21.93(S D=5.13)$. Approximately $88.8 \%$ of the sample was female. Additionally, $39.5 \%$ of the participants identified as non-Hispanic White, followed by 28.9\% Black/African American, 2.6\% AsianAmerican, $0.7 \%$ Native American and 9.2\% were others. Cultural self-identity of the participants included $40.4 \%$ individualists, $21.9 \%$ collectivists and $33.8 \%$ who did not declare their identity. Details about the study sample are presented in Table 1.

Table 1. Descriptive statistics for study sample $(N=154)$.

\begin{tabular}{lll}
\hline Study Variable & & Study Sample \\
\hline Age & & $21.93(5.13)$ \\
Gender & Male & $16(10.5)$ \\
& Female & $135(88.8)$ \\
Race & Black/African American & $29(19.1)$ \\
& Asian American & $4(2.6)$ \\
& Native American & $1(0.7)$ \\
& Latino/Latina & $44(28.9)$ \\
\hline
\end{tabular}


Table 1. Cont.

\begin{tabular}{|c|c|c|}
\hline Study Variable & & Study Sample \\
\hline & Non-Hispanic White & $60(39.5)$ \\
\hline & Other & $14(9.2)$ \\
\hline Education & & $3.08(1.28)$ \\
\hline Number of Family Members & & $3.95(1.80)$ \\
\hline \multirow[t]{2}{*}{ Single Parent Family } & Yes & $35(23.0)$ \\
\hline & No & $117(77.0)$ \\
\hline \multirow[t]{2}{*}{ Divorced Parents } & Yes & $48(31.6)$ \\
\hline & No & $104(68.4)$ \\
\hline \multirow[t]{3}{*}{ Living with Parents } & Yes-with one parent & $26(17.1)$ \\
\hline & Yes-with both parents & $55(36.2)$ \\
\hline & No & $71(46.7)$ \\
\hline \multirow{2}{*}{ Parents International Residency } & Yes & $15(9.9)$ \\
\hline & No & $137(90.1)$ \\
\hline Number of Siblings & & $2.27(2.12)$ \\
\hline \multirow[t]{3}{*}{ Cultural Identity } & Individualistic & $61(40.4)$ \\
\hline & Collectivistic & $33(21.9)$ \\
\hline & Do not know & $51(33.8)$ \\
\hline Individualism & & $6.06(1.30)$ \\
\hline Collectivism & & $6.90(1.26)$ \\
\hline Mobile Use & & $152(98.7)$ \\
\hline \multirow[t]{6}{*}{ Social Media Use } & Facebook & $117(76.0)$ \\
\hline & Instagram & $145(94.2)$ \\
\hline & Snapchat & 140 (90.9) \\
\hline & Twitter & $105(68.2)$ \\
\hline & Other & $11(7.1)$ \\
\hline & No social media & $3(1.9)$ \\
\hline Minutes Texting & & $80.60(78.61)$ \\
\hline Minutes Phone Call & & $42.17(58.31)$ \\
\hline Minutes Social Media & & 70.87 (94.69) \\
\hline
\end{tabular}

Note. Quantitative information is presented as the means with standard deviation in parentheses. Categorical information is presented as counts, with column percentages in parentheses.

\subsection{Procedure}

Professors teaching undergraduate human development and family sciences courses at a large university were invited to share an online survey with their classes that could be used as extra credit. Interested professors shared a link to an online survey with their students to complete, and each offered extra credit. When accessing the survey, participants were made aware of the qualifications, which were to be enrolled in an undergraduate human development and family science class at the university of study recruitment and be at least 18 years old. The online survey took approximately 30 minutes to complete. At the end of the online survey, participants were provided a link to direct them to a separate survey, where they could enter their information for extra credit, which allows for anonymity. All elements of this research project were approved by the appropriate institutional review board.

\subsection{Measures}

\section{Family Closeness}

Participants reported their closeness with up to six family members: two parents/ guardians, two grandparents (adoptive or biological), a sibling (if applicable), and an aunt or uncle (if applicable). Participants rated their closeness by multimodality, including texting, phone/virtual calls, direct messaging on social media, indirect communication on social media (such as liking, commenting, or sharing information), viewing information on social media, as well as their closeness spending time FtF. Participants answered two questions to indicate closeness per communication method: "How would you rate your closeness with (family member) through (type of communication)?", and "How would you 
rate your love for (family member) through (type of communication)?" Responses for each question ranged from 1 (very poor) to 7 (very good). Means and standard deviation for relationship quality for all relationships based on type of communication are presented in Table 2.

Table 2. Paired samples T-tests $(N=154)$.

\begin{tabular}{|c|c|c|c|}
\hline Parent $1(n=149)$ & Mean & $S D$ & $t$ \\
\hline Closeness via texting & 5.32 & 1.81 & $-7.65^{* * *}$ \\
\hline Closeness via voicecall & 6.03 & 1.66 & $-3.20 * *$ \\
\hline Closeness via direct social media & 5.57 & 2.55 & $-3.85^{* * *}$ \\
\hline Closeness via indirect social media & 5.69 & 2.42 & $-3.33^{* * *}$ \\
\hline Closeness via social media viewing & 5.62 & 2.30 & $-3.72 * * *$ \\
\hline Closeness via face to face & 6.43 & 1.24 & - \\
\hline Love via texting & 5.84 & 1.58 & $-5.09^{* * *}$ \\
\hline Love via voicecall & 6.25 & 1.62 & -1.60 \\
\hline Love via direct social media & 5.66 & 2.53 & $-3.52 * * *$ \\
\hline Love via indirect social media & 5.72 & 2.43 & $-3.21 * *$ \\
\hline Love via social media viewing & 5.80 & 2.18 & $-3.06^{* *}$ \\
\hline Love via face to face & 6.43 & 1.24 & - \\
\hline \multicolumn{4}{|l|}{ Parent $2(n=107)$} \\
\hline Closeness via texting & 5.28 & 2.04 & $-2.21 *$ \\
\hline Closeness via voicecall & 6.07 & 2.01 & 1.37 \\
\hline Closeness via direct social media & 6.31 & 2.56 & 1.98 \\
\hline Closeness via indirect social media & 6.22 & 2.56 & 1.65 \\
\hline Closeness via social media viewing & 7.00 & 1.94 & 3.02 \\
\hline Closeness via face to face & 5.74 & 1.68 & - \\
\hline Love via texting & 5.58 & 1.93 & -1.85 \\
\hline Love via voicecall & 6.16 & 2.02 & 0.883 \\
\hline Love via direct social media & 6.43 & 2.51 & 1.64 \\
\hline Love via indirect social media & 6.38 & 2.50 & 1.50 \\
\hline Love via social media viewing & 6.36 & 2.49 & 1.51 \\
\hline Love via face to face & 5.96 & 1.56 & - \\
\hline \multicolumn{4}{|l|}{ Grandparent $1(n=115)$} \\
\hline Closeness via texting & 5.93 & 2.43 & -0.675 \\
\hline Closeness via voicecall & 6.08 & 2.24 & 0.126 \\
\hline Closeness via direct social media & 6.38 & 2.73 & 1.339 \\
\hline Closeness via indirect social media & 6.22 & 2.74 & 0.642 \\
\hline Closeness via social media viewing & 6.11 & 2.74 & 0.210 \\
\hline Closeness via face to face & 6.05 & 1.76 & - \\
\hline Love via texting & 6.13 & 2.32 & -0.908 \\
\hline Love via voicecall & 6.24 & 2.23 & -0.331 \\
\hline Love via direct social media & 6.36 & 2.70 & 0.211 \\
\hline Love via indirect social media & 6.32 & 2.71 & 0.139 \\
\hline Love via social media viewing & 6.15 & 2.71 & -0.53 \\
\hline Love via face to face & 6.29 & 1.53 & - \\
\hline \multicolumn{4}{|l|}{ Grandparent $2(n=34)$} \\
\hline Closeness via texting & 6.30 & 2.52 & 0.50 \\
\hline Closeness via voicecall & 6.03 & 2.08 & 0.21 \\
\hline Closeness via direct social media & 7.21 & 2.01 & $2.48 *$ \\
\hline Closeness via indirect social media & 6.90 & 2.14 & 1.85 \\
\hline Closeness via social media viewing & 6.90 & 2.14 & 1.85 \\
\hline Closeness via face to face & 5.94 & 1.92 & - \\
\hline Love via texting & 6.63 & 2.31 & -0.07 \\
\hline Love via voicecall & 6.67 & 2.02 & 0.15 \\
\hline Love via direct social media & 7.21 & 2.01 & 1.16 \\
\hline Love via indirect social media & 7.21 & 2.01 & 1.18 \\
\hline Love via social media viewing & 6.97 & 2.04 & 0.68 \\
\hline Love via face to face & 6.55 & 1.42 & - \\
\hline
\end{tabular}


Table 2. Cont.

\begin{tabular}{|c|c|c|c|}
\hline Parent $1(n=149)$ & Mean & $S D$ & $t$ \\
\hline \multicolumn{4}{|l|}{ Sibling $(n=121)$} \\
\hline Closeness via texting & 6.02 & 1.23 & $-4.19^{* * *}$ \\
\hline Closeness via voicecall & 6.53 & 1.53 & 0.91 \\
\hline Closeness via direct social media & 6.26 & 1.95 & -0.79 \\
\hline Closeness via indirect social media & 6.61 & 1.84 & 1.08 \\
\hline Closeness via social media viewing & 6.21 & 1.92 & -1.10 \\
\hline Closeness via face to face & 6.40 & 1.13 & - \\
\hline Love via texting & 6.21 & 1.37 & $-3.90 * * *$ \\
\hline Love via voicecall & 6.70 & 1.44 & 0.86 \\
\hline Love via direct social media & 6.49 & 1.86 & -0.53 \\
\hline Love via indirect social media & 6.66 & 1.82 & 0.46 \\
\hline Love via social media viewing & 6.35 & 1.89 & -1.44 \\
\hline Love via face to face & 6.60 & 1.02 & - \\
\hline \multicolumn{4}{|l|}{ Aunt/Uncle $(n=52)$} \\
\hline Closeness via texting & 6.02 & 1.93 & -0.08 \\
\hline Closeness via voicecall & 6.50 & 1.92 & $2.02 *$ \\
\hline Closeness via direct social media & 6.92 & 2.07 & $3.17 * *$ \\
\hline Closeness via indirect social media & 6.53 & 2.23 & 1.55 \\
\hline Closeness via social media viewing & 5.90 & 2.41 & -0.519 \\
\hline Closeness via face to face & 6.06 & 1.35 & - \\
\hline Love via texting & 6.17 & 1.90 & -0.21 \\
\hline Love via voicecall & 6.50 & 2.04 & 0.96 \\
\hline Love via direct social media & 7.04 & 1.96 & $2.58 * *$ \\
\hline Love via indirect social media & 6.76 & 2.02 & 1.15 \\
\hline Love via social media viewing & 5.96 & 2.48 & -1.09 \\
\hline Love via face to face & 6.25 & 1.41 & - \\
\hline
\end{tabular}

\section{Multimodality and FtF Behaviors}

Participants answered two questions regarding six different types of communication: texting, virtual/phone calls, direct messaging on social media, indirect communication on social media, viewing information on social media, and spending time FtF. These questions included, "How frequently do you [communication method; i.e., text] this (family member)?" and "How long do these interactions commonly last (in minutes)?" Responses ranged from 0 (never) to 8 (daily). Means and standard deviations for these variables are also presented in Table 2.

\section{Collectivism}

This scale was measured using the 8-item collectivism subscale by Triandis and Gelfand (1998). Example items include, "I feel good when I cooperate with others" and "Parents and children must stay together as much as possible." Responses ranged from 1 (never or definitely no) to 9 (always or definitely yes) and this scale demonstrated adequate internal consistency (Cronbach's alpha $=0.82)$.

\section{Data Analysis}

To address the first hypothesis, which stated that spending time FtF would be more strongly associated with relationship closeness and love than interacting through technology and media, paired sample $t$-tests were conducted, comparing the means of spending time FTF with the means of communicating through other approaches. These analyses are conducted across all relationships: parent one, parent two, grandparent one, grandparent two, sibling, and aunt or uncle. Next, correlational analyses are conducted using all communication methods and relationship closeness and love for each family type. To address the second hypothesis, which states that collectivism will moderate the relationship between communication approach and relationship quality, regression analyses will be conducted. To conduct moderation analyses, we follow the guidelines provided by 
(Aiken and West 1991). For this analyses, the types of communication (texting, voice calling, direct messaging, indirect messaging, viewing social media, and FtF) and scores of collectivism will be mean centered and included as predictor variables for relationship closeness and love across all six different relationships captured in this study. To test for moderation, the interaction between type of communication and collectivism will be included in these analyses.

\section{Results}

First, paired sample $t$-tests were conducted to evaluate whether spending time FtF with family members was more closely aligned with relationship quality than multimodality. Results of these analyses are presented in Table 2. We found some support for our hypothesis for the relationship between participants and parent one. The mean of FtF closeness for parent one was significantly higher compared to all measures of multimodality. The mean of FtF love for parent one was also statistically higher than all types of multimodality, except voice calling. However, we did not receive confirmation of our hypothesis with parent two, as only one significant difference was found; the mean for texting was significantly lower than the mean for FtF for relationship closeness. No other means were significantly different for spending time FtF. There was also little support for our hypothesis with grandparents. For grandparent one, no means were significantly different from spending time $\mathrm{FtF}$ for closeness or love. Interestingly, for grandparent two, spending time on social media was rated as significantly higher for relationship closeness than spending time FtF. There was partial support for sibling love and closeness as texting was rated as significantly lower than spending time FtF, but there were no other differences for the other multimodalities. For aunt or uncle, we found support counter to our hypothesis, as spending time direct messaging on social media was rated as higher compared to spending time $\mathrm{FtF}$ for love and closeness and voice calling was associated with higher closeness compared to spending time FtF.

Next, we examined correlations across each of our dependent variables as well as collectivism and individualism. We found that collectivism was positively associated with closeness and love from texting $(r=0.31 ; p<0.01 ; r=0.44 ; p<0.01)$, voice calling $(r=0.40$; $p<0.01 ; r=0.38 ; p<0.01)$, and spending time $\mathrm{FtF}(r=0.40 ; p<0.01 ; r=0.39 ; p<0.01)$ with parent one. Collectivism was also positively associated with love and closeness from texting and video calls with parent two $(r=0.24 ; p<0.05 ; r=0.24 ; p<0.05 ; r=0.21 ; p<0.05$; $r=0.25 ; p<0.05$, respectively). For both parent one and parent two, love from texting and spending time $\mathrm{FtF}$ was positively associated with individualism $(r=0.17 ; p<0.05$; $r=0.17 ; p<0.05 ; r=0.31 ; p<0.01 ; r=0.44 ; p<0.01$, respectively). Love from texting was negatively associated with individualism $(r=-0.19 ; p<0.05)$, and love from spending time FtF was positively associated with collectivism $(r=0.19 ; p<0.05)$ with grandparent one. Collectivism and individualism were not positively associated with love or closeness from any multimodality or spending time $\mathrm{FtF}$ with grandparent two. Closeness from spending time FtF with participant's sibling was positively associated with collectivism $(r=0.22$; $p<0.05)$, but individualism was not associated with any of the dependent variables. Last, individualism and collectivism were not associated with any of the dependent variables.

Through the correlation analyses, we received some indication that collectivism could moderate the relationship between multimodality (including FtF) and relationship closeness. The results of this analyses are presented in Table 3. Based on these results, we find partial support for the moderating role of collectivism across multiple family relationships. For example collectivism moderated the relationship between texting and spending time $\mathrm{FtF}$ and relationship quality with parent one, where those high in collectivism displayed higher closeness with texting and FtF compared to those who were lower in collectivism, particularly when use of texting and time spent $\mathrm{FtF}$ was high. Results of these analyses are presented in Figures 1 and 2. However, no other significant moderating effect was found across other family relationships. 
Table 3. Moderation analyses of collectivism for multimodality, $\mathrm{FtF}$ and family closeness.

\begin{tabular}{|c|c|c|c|c|}
\hline & \multicolumn{2}{|c|}{ Parent 1} & \multicolumn{2}{|c|}{ Parent 2} \\
\hline & Closeness & Love & Closeness & Love \\
\hline \multicolumn{5}{|l|}{ Model 1} \\
\hline Texting & $0.93(0.34)^{* * *}$ & $10.00(0.28)^{* * *}$ & $0.96(0.39)$ & $0.98(0.37) *$ \\
\hline Collectivism & $0.77(0.35)^{* * *}$ & $10.02(0.29)^{* * *}$ & $0.63(0.36)^{* *}$ & $0.63(0.34)^{* *}$ \\
\hline Texting $\times$ Collectivism & $0.91(0.05) *$ & $10.09(0.04)^{* * *}$ & $-0.99(0.06)$ & $-0.97(0.05)$ \\
\hline \multicolumn{5}{|l|}{ Model 2} \\
\hline Voicecalling & $-0.18(0.26)$ & $-0.06(0.26)$ & $-0.87(0.34)$ & $-0.85(0.34)$ \\
\hline Collectivism & $20.32(0.40)$ & $0.40(0.23) *$ & $-0.23(0.28)$ & $-0.23(0.28)$ \\
\hline Voicecalling $\times$ Collectivism & $0.04(0.04)$ & $-0.05(0.04)$ & $0.87(0.05)$ & $0.87(0.05)$ \\
\hline \multicolumn{5}{|l|}{ Model 3} \\
\hline Directmessaging & $-0.12(0.37)$ & $-0.44(0.41)$ & $-0.36(0.23)$ & $-0.20(0.24)$ \\
\hline Collectivism & $0.12(0.22)$ & $0.09(0.22)$ & $-0.50(0.35)$ & $-0.48(0.36)^{*}$ \\
\hline Directmessaging $\times$ Collectivism & $0.01(0.05)$ & $0.36(0.06)$ & $0.09(0.07)$ & $-0.07(0.07)$ \\
\hline \multicolumn{5}{|l|}{ Model 4} \\
\hline Indirectmessaging & $0.01(0.32)$ & $-0.10(0.32)$ & $-0.44(0.39)$ & $-0.37(0.38)$ \\
\hline Collectivism & $0.13(0.22)$ & $0.13(0.22)$ & $-0.12(0.23)$ & $-0.12(0.23)$ \\
\hline Indirectmessaging $\times$ Collectivism & $-0.12(0.05)$ & $-0.02(0.05)$ & $0.34(0.06)$ & $0.32(0.05)$ \\
\hline \multicolumn{5}{|l|}{ Model 5} \\
\hline Viewing & $-10.1(0.31)^{*}$ & $-0.97(0.30)^{*}$ & $-0.34(0.45)$ & $-0.22(0.45)$ \\
\hline Collectivism & $-0.11(0.22)$ & $-0.06(0.21)$ & $-0.06(0.22)$ & $-0.08(0.22)$ \\
\hline Viewing $\times$ Collectivism & $0.97(0.04) *$ & $0.75(0.04)$ & $0.13(0.07)$ & $0.06(0.06)$ \\
\hline \multicolumn{5}{|l|}{ Model 6} \\
\hline Face to face & $10.65(0.25)^{* * *}$ & $10.60(0.23)^{* * *}$ & $0.67(0.34)$ & $0.60(0.32)$ \\
\hline Collectivism & $10.14(0.21)^{* * *}$ & $10.12(0.20)^{* * *}$ & $0.31(0.27)$ & $0.32(0.25)$ \\
\hline \multirow[t]{3}{*}{ Face to face $\times$ Collectivism } & $10.69(0.04)^{* * *}$ & $10.65(0.04)^{* * *}$ & $0.32(0.05)$ & $-0.28(0.05)$ \\
\hline & \multicolumn{2}{|c|}{ Grandparent1 } & \multicolumn{2}{|c|}{ Grandparent2 } \\
\hline & Closeness & Love & Closeness & Love \\
\hline \multicolumn{5}{|l|}{ Model 1} \\
\hline Texting & $-0.33(0.44)$ & $-0.36(0.42)$ & $-0.17(0.80)$ & $0.11(0.73)$ \\
\hline Collectivism & $-0.10(0.31)$ & $-0.08(0.30)$ & $-0.09(0.57)$ & $0.06(0.52)$ \\
\hline Texting $\times$ Collectivism & $0.34(0.06)$ & $0.41(0.06)$ & $0.25(0.12)$ & $0.05(0.11)$ \\
\hline \multicolumn{5}{|l|}{ Model 2} \\
\hline Voicecalling & $0.32(0.46)$ & $0.42(0.46)$ & $-0.08(0.68)$ & $-0.01(0.60)$ \\
\hline Collectivism & $0.07(0.27)$ & $0.07(0.27)$ & $-0.22(0.43)$ & $-0.18(0.38)$ \\
\hline Voicecalling $\times$ Collectivism & $-0.37(0.07)$ & $-0.43(0.07)$ & $0.07(0.10)$ & $0.05(0.09)$ \\
\hline \multicolumn{5}{|l|}{ Model 3} \\
\hline Directmessaging & $0.14(0.69)$ & $-0.14(0.68)$ & $-0.37(0.82)$ & $-0.37(0.83)$ \\
\hline Collectivism & $-0.05(0.27)$ & $-0.05(0.26)$ & $-0.12(0.36)$ & $-0.12(0.36)$ \\
\hline Directmessaging $\times$ Collectivism & $-0.22(0.10)$ & $0.04(0.10)$ & $0.18(0.17)$ & $0.18(0.17)$ \\
\hline \multicolumn{5}{|l|}{ Model 4} \\
\hline Indirectmessaging & $0.20(0.70)$ & $0.03(0.70)$ & $-0.17(0.89)$ & $-0.17(0.89)$ \\
\hline Collectivism & $0.05(0.27)$ & $0.04(0.27)$ & $-0.01(0.37)$ & $-0.01(0.37)$ \\
\hline
\end{tabular}


Table 3. Cont.

\begin{tabular}{|c|c|c|c|c|}
\hline Indirectmessaging $\times$ Collectivism & $-0.36(0.10)$ & $-0.08(0.10)$ & $0.13(0.17)$ & $0.13(0.17)$ \\
\hline \multicolumn{5}{|l|}{ Model 5} \\
\hline Viewing & $-0.24(0.74)$ & $-0.08(0.73)$ & $-0.34(0.80)$ & $-0.20(0.77)$ \\
\hline Collectivism & $0.02(0.28)$ & $-0.01(0.28)$ & $0.08(0.46)$ & $0.09(0.43)$ \\
\hline Viewing $\times$ Collectivism & $0.17(0.11)$ & $0.01(0.11)$ & $0.21(0.14)$ & $0.14(0.13)$ \\
\hline \multicolumn{5}{|l|}{ Model 6} \\
\hline Face to face & $-0.28(0.45)$ & $-0.22(0.39)$ & $-20.42(10.28)$ & $-0.45(10.00)$ \\
\hline Collectivism & $0.13(0.27)$ & $0.11(0.23)$ & $-0.65(0.69)$ & $-0.06(0.55)$ \\
\hline \multirow[t]{3}{*}{ Face to face $\times$ Collectivism } & $0.26(0.07)$ & $0.25(0.06)$ & $20.35(0.18)$ & $0.41(0.14)$ \\
\hline & \multicolumn{2}{|c|}{ Sibling } & \multicolumn{2}{|c|}{ Aunt/Uncle } \\
\hline & Love & Closeness & Love & Love \\
\hline \multicolumn{5}{|l|}{ Model 1} \\
\hline Texting & $0.64(0.47)$ & $-0.29(0.64)$ & $-0.35(0.64)$ & $0.11(0.73)$ \\
\hline Collectivism & $0.35(0.49)$ & $-0.00(0.29)$ & $-0.00(0.28)$ & $0.06(0.52)$ \\
\hline Texting $\times$ Collectivism & $-0.64(0.07)$ & $0.06(0.09)$ & $0.18(0.09)$ & $0.05(0.11)$ \\
\hline \multicolumn{5}{|l|}{ Model 2} \\
\hline Voicecalling & $-0.23(0.24)$ & $-10.25(0.69)$ & $-10.27(0.75)$ & $-0.01(0.60)$ \\
\hline Collectivism & $0.13(0.17)$ & $0.02(0.27)$ & $0.02(0.29)$ & $-0.18(0.38)$ \\
\hline Voicecalling $\times$ Collectivism & $-0.01(0.03)$ & $0.98(0.10)$ & $10.04(0.11)$ & $0.05(0.09)$ \\
\hline \multicolumn{5}{|l|}{ Model 3} \\
\hline Directmessaging & $-0.47(0.32)$ & $0.48(0.80)$ & $0.12(0.78)$ & $-0.37(0.83)$ \\
\hline Collectivism & $0.01(0.23)$ & $0.04(0.26)$ & $0.05(0.26)$ & $-0.12(0.36)$ \\
\hline Directmessaging $\times$ Collectivism & $0.31(0.05)$ & $-0.77(0.11)$ & $-0.34(0.11)$ & $0.18(0.17)$ \\
\hline \multicolumn{5}{|l|}{ Model 4} \\
\hline Indirectmessaging & $-0.14(0.28)$ & $-0.48(0.73)$ & $-0.03(0.66)$ & $-0.17(0.89)$ \\
\hline Collectivism & $0.13(0.18)$ & $0.26(0.29)$ & $0.29(0.26)$ & $-0.01(0.37)$ \\
\hline Indirectmessaging $\times$ Collectivism & $-0.23(0.04)$ & $0.17(0.10)$ & $-0.28(0.09)$ & $0.13(0.17)$ \\
\hline \multicolumn{5}{|l|}{ Model 5} \\
\hline Viewing & $-0.47(0.08)^{* * *}$ & $-10.12(65)$ & $-0.95(0.68)$ & $-0.20(0.77)$ \\
\hline Collectivism & $0.38(0.20)^{* * *}$ & $0.15(0.36)$ & $0.15(0.38)$ & $0.09(0.43)$ \\
\hline Viewing $\times$ Collectivism & $-0.10(0.01)$ & $0.74(0.09)$ & $0.60(0.10)$ & $0.14(0.13)$ \\
\hline \multicolumn{5}{|l|}{ Model 6} \\
\hline Face to face & $0.57(0.31)$ & $-20.36(0.50)$ & $-10.10(0.56)$ & $-0.45(10.00)$ \\
\hline Collectivism & $0.44(0.28)$ & $-0.28(0.24)$ & $-0.27(0.27)$ & $-0.06(0.55)$ \\
\hline Face to face $\times$ Collectivism & $-0.65(0.05)$ & $20.24(0.07)$ & $10.93(0.08)$ & $0.41(0.14)$ \\
\hline
\end{tabular}

$$
\text { *** } p<0.001 ;{ }^{* *} p<0.01 ; p<0.05 \text {. }
$$




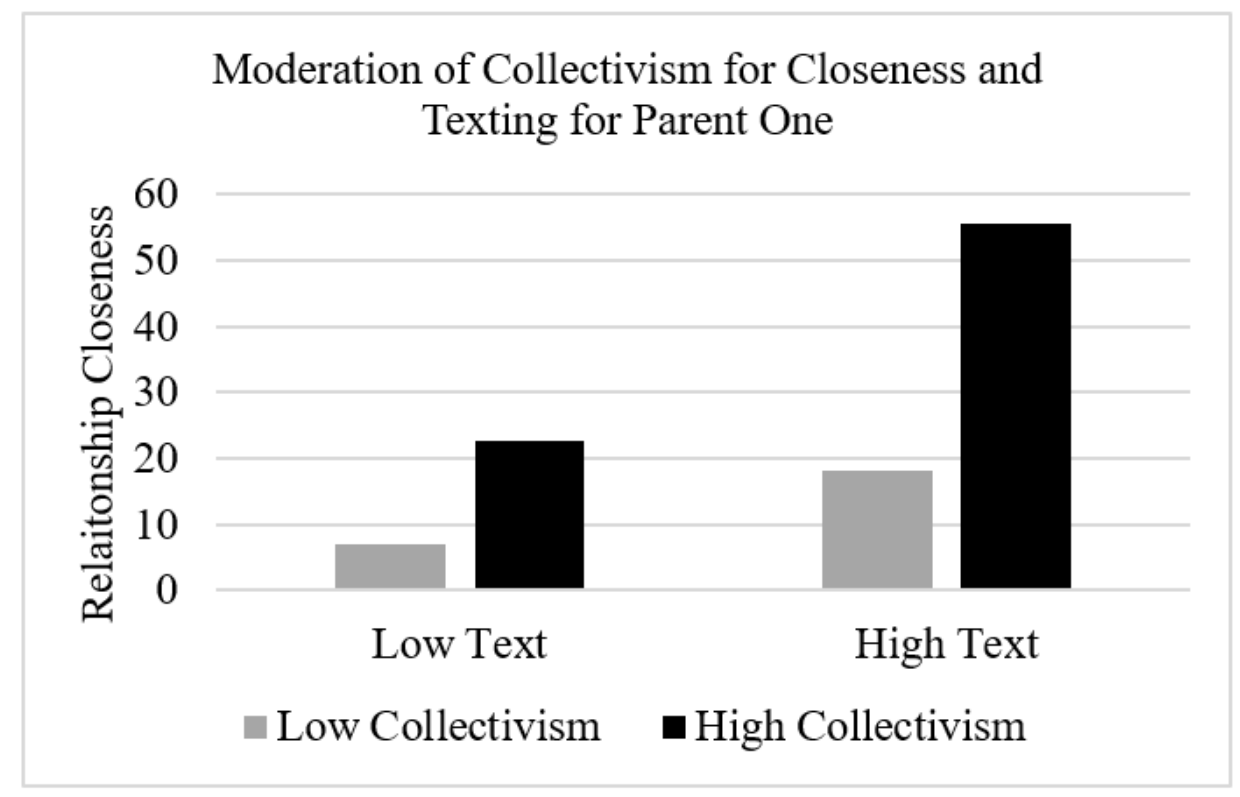

Figure 1. This figure displays how collectivism moderated the relationship between closeness and texting for parent one. Those who texted more often reported higher closeness with their parent, and family closeness was high even if individuals identified as high in collectivism.

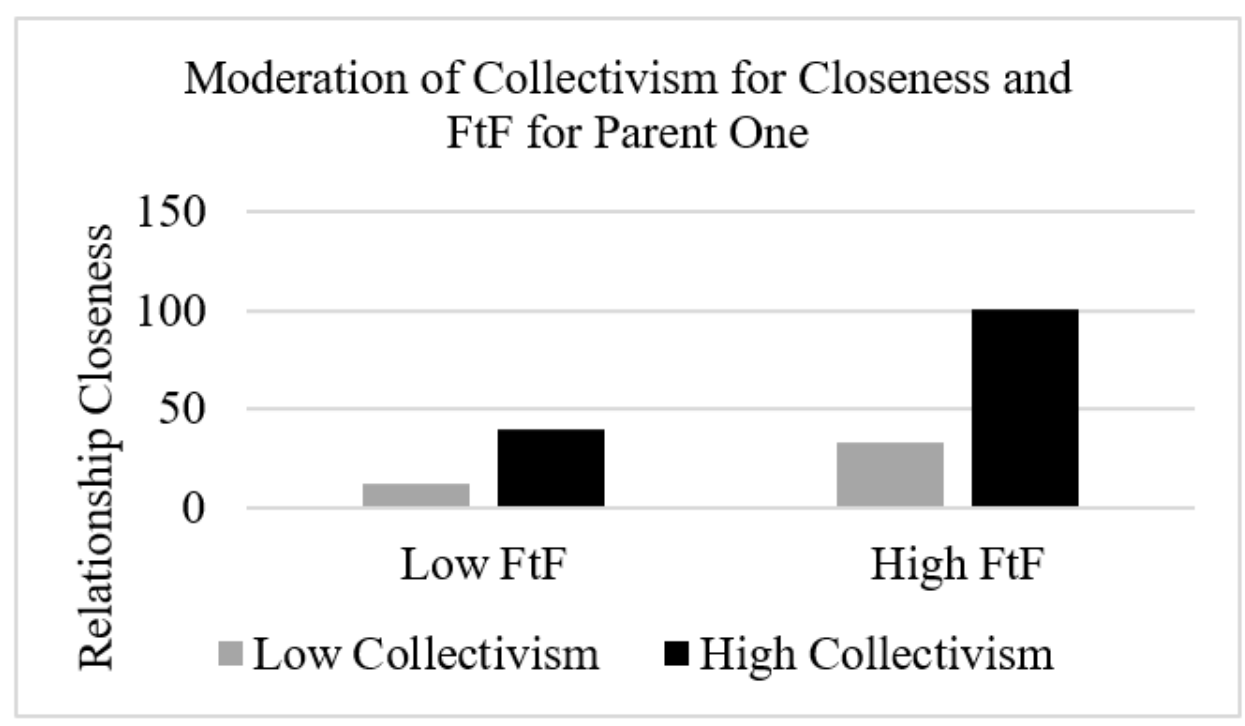

Figure 2. This graph depicts the moderating effect of collectivism for time spent FtF and relationship closeness. Having high levels of collectivism was associated with more family closeness, but this was especially true for those who spent more FtF time with parent one.

\section{Discussion}

Based on study results, spending time FtF was linked to higher-quality parental relationships compared to multimodality for young adults. Yet, connecting with grandparents, siblings, aunts, and uncles through multimodalities was also correlated with high levels of closeness. When examining collectivism as a moderator for these relationships, spending time FtF with parent one was moderated by collectivism, but no other significant moderating relationships were found in this study. The benefits of spending time FtF appear to be important for relationships with family members that they are already close to, such as one's parent(s), and this is particularly true if someone identifies as collectivistic. These findings challenge some of the main tenets of MMT with parental relationships but provide support for increasing closeness with other family relationships. 
First, individuals appear to have stronger relationships with their parents when they engage in offline behaviors compared to online behaviors. This finding contradicts MMT, which presumes that $\mathrm{FtF}$ time is equivalent to time spent communicating virtually. It is likely that the parental relationship is one of the closest relationships that an individual has with someone. In the case of parents, individuals may be used to seeing them in person and subsequently want to maintain this relationship in the same way they are used to. More precisely, individuals often live with their parents growing up, and although many leave the home shortly after adolescence, many individuals may seek to grow that relationship through $\mathrm{FtF}$ as it reminds them of their relationship growing up. In other words, as one grows up by interacting with their parent FtF, spending time FtF may be one of the better ways to increase relationship quality as online behaviors were less relied on growing up.

Consistent with previous studies, spending time FtF provides more opportunity to develop and maintain interdependence through mutual self-disclosure. Several studies illustrate how FtF communication is important for relationship maintenance (Jamieson et al. 2020; Laurenceau et al. 2004; Sproull et al. 1986). Communication is likely to be more reciprocal and effective when communicating FtF, as opposed to communicating online, given nonverbal cues and facial expressions (Sherman et al. 2013). Consistent with previous studies that examined mutual self-disclosure in intimate relationships, online interactions were rated less as less meaningful as interactions that were communicated offline (Jamieson et al. 2020). Given the intimate relationship between individuals and their parents, the finding that FtF predicted increased closeness compared to multimodality is not surprising given the literature on the importance of offline behaviors for close relationships.

This finding is even more important for those who already view the family as their central focus, meaning that they have a collectivistic cultural orientation. Individuals who support their family over themselves appear to prefer FtF interactions with their parents over and beyond online behaviors (besides texting). This finding adds to the results of Barakji et al. (2018), who found that increased communication, particularly via multimodality, brings families high in collectivism closer together. The current study found that online behaviors were related to relationship closeness and love, but FtF contributed to higher levels of relationship quality. Significant interactions in the current study show that closeness is highest with one's parent when collectivism and spending time FtF is high. For collectivistic cultures, the ability to communicate reciprocally and provide support in person aligns with the central tenets of collectivism. It is more difficult to provide this support virtually, especially with family members that you are likely to be especially close with (i.e., parents).

Despite the strong support for FtF and relationship closeness with a parent, less support was found for the importance of $\mathrm{FtF}$ with other family relationships. Results demonstrated that multimodality predicted closeness similar to time spent FtF with grandparents, siblings, aunts, and uncles. This finding remained consistent even when testing collectivism as a moderator. These findings support MMT, as increased multimodalities were beneficial for these family relationships. According to this theory, the increased multimodality we have with someone, the stronger the relationship quality. For these family relationships, this concept remains true. If an individual wants to be closer with a family member (who is not their parent), connecting online provides an easy, viable option to communicate, become updated on each other's lives, and become closer with each other.

The use of media with family members also shows an evolution of how young adults communicate with family members. Since it is likely that individuals may not live or see their grandparents, siblings (particularly if they are in college), aunts, and uncles on a regular basis, the best way to maintain these family relationships may be through online communication. Online behaviors do not require much effort or investment, which makes communicating easier, providing support for why FtF did not contribute more to relationship quality than offline behaviors. It may be that individuals are simply used to "spending time" with these family members online. The affordances provided by technology make communication with others, including family members, easier. Rather than trying to 
physically visit with a family member, someone can easily text them, video call them, or view their social media profile. The ability to connect with someone via multimodality provides more opportunities to connect with family members that we may not be able to see regularly or consistently. For the current study, online and offline behaviors both contribute to relationship closeness with multiple extended family members.

Results from this study provide some empirical evidence for optimizing family closeness. First, if we want a closer family relationship with our closest parent, we should maximize our $\mathrm{FtF}$ connection. Due to the significant association between spending time $\mathrm{FtF}$ and family closeness, establishing a FtF connection with a close parent may reduce family conflict. Results of the current study also illustrate that connecting with other family members online may be just as helpful as spending time FtF. It may be more difficult to see grandparents, aunts, and uncles presumably because of geographical distance. Thus, the current study shows some benefits of connecting with these family members through social media, at least for the quality of these family relationships. This study also has implications for MMT, which advocates for multimodality for relationship quality, as multimodality views online and offline behaviors as relatively equal. Although this appears true with extended family relationships, these results do not appear consistent with one's parent. MMT is based on group processes (Haythornthwaite 2002), which are not necessarily intimate and close relationships. The relationships in which individuals view as highly interdependent, spending time FtF may be best to maintain those relationships. Relationships with family members that we may not see or talk to often may benefit most with multimodality and FtF. Generally, the central premise of MMT may hold true for close relationships, but maybe not for our closest relationships, assuming that the parental relationship is particularly close.

In addition to addressing family relationships, the results of this study have theoretical implications. These studies appear supportive and also critical of MMT, particularly the importance of FtF interaction. Subsequently, this theory could be improved by incorporating cultural dimensions, such as collectivism and individualism. It may also be reasonable to establish a new theory that integrates not only culture and media connections, but also FtF interactions, particularly in ways that help strengthen relationships. Generally, this study extends the scope of MMT by examining FtF interactions and cultural perspectives in family relationship and closeness.

This study was conducted before the COVID-19 pandemic. Despite this timing, these results have a significant contribution to understanding how relying on technology for communication can impact those relationships. There are certainly some affordances of social media and technology that allows communication between family members, particularly during a time when $\mathrm{FtF}$ is not possible. However, physical and social distancing as well as isolation with family members during the COVID-19 period may prompt loneliness among the members, particularly strong family relationships, such as the relationship with one's closest parent. The inability to spend time FtF could potentially hinder mental health (Koh and Liew 2020). Thus, the findings of this study show that technology can be good for relationships, but an over-reliance on technology could take a toll on the quality of these relationships, which could also have an impact on individual well-being. In this regard, further studies are needed to contextualize these findings within the COVID-19 pandemic.

\section{Limitations and Conclusions}

While this study advances the debate on online versus offline behaviors for relationship quality and MMT, several limitations are present. Most notable was that the sample was not generalizable based on age (mostly young adults), gender (mostly female), and education (all college students). This population is known for using social media more consistently and regularly than other populations. Thus, the generalizability of the results is limited. Second, the number of participants reporting on extended family relationships was lower compared to parental relationships, which decreases analytic power. The methodology of this study, which is primarily regression and correlational, are not stringent enough to determine cause and effect. Although we claim that spending time FtF is associated 
with more closeness with parents, it may be just as likely that when people are close with their parent, they tend to spend more time FtF. The goal of the current study was to better understand the contribution of online and offline behaviors for family relationships and should be viewed as a starting point with these variables. Future studies should explore these relationships dyadically-do parents, grandparents, siblings, aunts, and uncles also feel close with young adults through multimodality and spending time FtF? Measuring these variables longitudinally would also be helpful, as multimodality and spending time FtF may vary over extended periods of time. By conducting longitudinal and dyadic research, stronger, more valid claims can be made about how to optimally promote family relationship quality.

Despite these limitations, this study advanced the literature in a number of ways. First, this study examined a multitude of family relationships that have not received a fair amount of empirical investigation. Second, this study not only examined social media use in family relationships, but specifically examined different behaviors on social media to best understand how online and offline behaviors contribute to relationship closeness. This study also capitalized on previous research by examining the role of collectivism as a moderator. Through these approaches, the current study illustrates the importance of spending time FtF with one's closest parent, but also shows that multimodality can be just as important for the closeness with one's extended family. Future studies should explore these relationships longitudinally and dyadically to gain a stronger comprehension of the contribution of online and offline behaviors for family relationships. In conclusion, the findings of this study highlight the potential impact of FtF in the context of COVID-19, providing a direction for post-COVID-19 studies.

Author Contributions: This study was designed jointly by B.B. and M.L. Data curation, formal analysis, investigation, methodology, project administration and writing-original draft report was done by B.B. with close supervision by M.L.; and writing-review \& editing parts were done by M.L. All authors have read and agreed to the published version of the manuscript.

Funding: The author(s) received no external financial support.

Institutional Review Board Statement: The study was conducted according to the guidelines of the Declaration of Helsinki and approved by the Institutional Review Board of the University of North Texas (IRB-20-266).

Informed Consent Statement: Informed consent was obtained from all subjects involved in the study.

Data Availability Statement: Data is available upon request from the first author.

Conflicts of Interest: The authors declare no conflict of interest.

\section{References}

Aiken, Leona S., and Stephen G. West. 1991. Multiple Regression: Testing and Interpreting Interactions. Thousand Oaks: Sage Publications, Inc. Anderson, Monica. 2019. Internet and Technology. Mobile Technology and Home Broadband 2019. Available online: https: / / www.pewresearch.org/internet/2019/06/13/mobile-technology-and-home-broadband-2019/ (accessed on 13 June 2019).

Barakji, Fatima, Katheryn C. Maguire, Holly Reiss, Jaclyn Gaule, Nicholas Smith, Lukas Pelliccio, Scott Sellnow-Richmond, Jehoon Jeon, and Hayg Oshagan. 2018. Cultural and Transnational Influences on the Use of Information Communication Technologies in Adult Long-Distance Family Relationships: An Extension of Media Multiplexity Theory. Journal of Family Communication 19: 30-46. [CrossRef]

Clement, J. 2019. Mobile Social Media-Statistics \& Facts. Statista. Available online: https://www.statista.com/topics/2478/mobilesocial-networks / (accessed on 15 December 2019).

Dutot, Vincent. 2020. A Social Identity Perspective of Social Media's Impact on Satisfaction with Life. Psychology E Marketing 37: 759-72. [CrossRef]

Ellison, Nicole B., Charles Steinfield, and Cliff Lampe. 2007. The Benefits of Facebook 'Friends:' Social Capital and College Students' Use of Online Social Network Sites. Journal of Computer-Mediated Communication 12: 1143-68. [CrossRef]

Finkel, Eli J., Eastwick Paul W., Karney Benjamin R., Reis Harry T., and Sprecher Susan. 2012. Online Dating: A Critical Analysis from the Perspective of Psychological Science. Psychological Science in the Public Interest 13: 3-66. [CrossRef] [PubMed] 
Hamamura, Takeshi. 2012. Are Cultures Becoming Individualistic? A Cross-Temporal Comparison of Individualism-collectivism in the United States and Japan. Personality and Social Psychology Review 16: 3-24. [CrossRef] [PubMed]

Haythornthwaite, Caroline. 2002. Building Social Networks via Computer Networks: Creating and Sustaining Distributed Learning Communities. In Building Virtual Communities: Learning and Change in Cyberspace. Edited by K. Ann Renninger and Wesley Shumar. New York: Cambridge University Press, pp. 169-90. [CrossRef]

Haythornthwaite, Caroline. 2005. Social Networks and Internet Connectivity Effects. Information, Communication E Society 8: 125-47. [CrossRef]

Hofstede, Greert. 2001. Culture's Consequences: Comparing Values, Behaviors, Institutions, and Organizations across Nations, 2nd ed. Thousand Oaks: Sage.

Jamieson, Jack, Boase Jeffrey, and Kobayashi Tetsuro. 2020. Multiplying the Medium: Tie Strength, Social Role, and Mobile Media Multiplexity. In The Handbook of Communication in the Networked Age. Oxford: Oxford University Press.

Kennedy, Tracy L. M., Smith Aaron, Wells Amy T., and Wellman Barry. 2008. Networked Families. Pew Internet \& American Life Project. Available online: https://www.pewresearch.org/internet/wp-content/uploads/sites/9/media/Files/Reports/2008 /PIP_Networked_Family.pdf.pdf (accessed on 19 October 2008).

Koh, Jing Xuan, and Tau Ming Liew. 2020. How loneliness is talked about in social media during covid-19 pandemic: Text mining of 4492 twitter feeds. Journal of Psychiatric Research. [CrossRef] [PubMed]

Laurenceau, Jean-Philippe, Lisa Feldman Barrett, and Paula R. Pietromonaco. 2004. Intimacy as an Interpersonal Process: The Importance of Self-Disclosure, Partner Disclosure, and Perceived Partner Responsiveness in Interpersonal Exchanges. In Close Relationships: Key Readings. Edited by Harry T. Reis and Caryl E. Rusbult. Philadelphia: Taylor \& Francis, pp. $199-211$.

Ledbetter, Andrew M. 2015. Media Multiplexity Theory: Technology Use and Interpersonal Tie Strength. In Engaging Theories in Interpersonal Communication, 2nd ed. Edited by Dawn O. Braithwaite and Paul Schrodt. Thousand Oaks: Sage, pp. 357-70.

Mesch, Gustavo S. 2006. Family Relations and the Internet: Exploring a Family Boundaries Approach. Journal of Family Communication 6: 119-38. [CrossRef]

Perrin, Andrew, and Monica Anderson. 2019. Share of U.S. Adults using Social Media, Including Facebook, Is Mostly Unchanged Since 2018: Pew Research Center. Available online: https://www.pewresearch.org/fact-tank/2019/04/10/share-of-u-s-adults-usingsocial-media-including-facebook-is-mostly-unchanged-since-2018/ (accessed on 10 April 2019).

Pew Research Center. 2019. Internet and Technology. Social Media Fact Sheet. Available online: https://www.pewresearch.org/ internet/fact-sheet/social-media/ (accessed on 12 June 2019).

Ruppel, Erin K., Tricia J. Burke, and Maura R. Cherney. 2018. Channel Complementarity and Multiplexity in Long-Distance Friends' Patterns of Communication Technology Use. New Media \& Society 20: 1564-79. [CrossRef]

Sherman, Lauren E., Michikyan Minas, and Greenfield Patricia M. 2013. The Effects of Text, Audio, and In-person Communication on Bonding Between Friends. Cyberpsychology: Journal of Psychosocial Research on Cyberspace. [CrossRef]

Sproull, Lee, David Zubrow, and Sara Kiesler. 1986. Cultural Socialization to Computing in College. Computers in Human Behavior 2: 257-75. [CrossRef]

Triandis, Harry C., and Michele J. Gelfand. 1998. Converging Measurement of Horizontal and Vertical Individualism and Collectivism. Journal of Personality and Social Psychology 74: 118-28. [CrossRef]

United Nations: Department of Economic and Social Affairs. 2019. International Migration: Report. Available online: https: //www.un.org/en/development/desa/population/migration/publications/migrationreport/docs/InternationalMigration2 019_Report.pdf (accessed on 12 June 2019).

Valkenburg, Patti M., and Jochen Peter. 2011. Online Communication among Adolescents: An Integrated Model of Its Attraction, Opportunities, and Risks. Journal of Adolescent Health 48: 121-27. [CrossRef] [PubMed]

Williams, Amanda L., and Michael J. Merten. 2011. IFamily: Internet and Social Media Technology in the Family Context. Family and Consumer Sciences Research Journal 40: 150-70. [CrossRef] 